\title{
A New Extension of Quasi Lindley Distribution: Properties and Applications
}

\author{
Unyime Patrick Udoudo $^{1 *}$ and Ette Harrison Etuk ${ }^{2}$ \\ ${ }^{1}$ Department of Mathematics, Rivers State University, Port Harcourt, Nigeria \\ ${ }^{2}$ Department of Mathematics, Rivers State University, Port Harcourt, Nigeria \\ *Corresponding author E-mail:udoudogeno@gmail.com
}

\begin{abstract}
In this paper, we introduced and studied the statistical properties of a new distribution called the Marshall-Olkin extended quasi Lindley distribution. Specifically, we derived the crude moment, moment generating function, quantile function, and distributions of order statistics based on the distribution. The maximum likelihood point estimation method was used to estimate the parameters of the newly introduced model. Some AR minfication processes were discussed. We illustrated the applicability of the distribution using a real dataset.
\end{abstract}

Keywords: Marshal-Olkin family of distributions; maximum likelihood estimates; minification processes; quasi lindley distribution; quantile function.

\section{Introduction}

Probability distributions are quite indispensable in many data analysis situations. In spite of numerous distributions in the literature, it has become a matter of necessity to generalise certain distributions so as to introduce relatively more flexible distributions. By introducing an additional parameter into a given distribution, especially a shape parameter, it is possible to improve upon the flexibility of the distribution. When one or more parameters have been introduced into a baseline distribution, the distribution is said to be extended or generalised.

The Lindley distribution (LD) introduced by Lindley(1958) is one of the widely used distributions. This distribution has been generalised in previous studies using several methods of adding parameters to distributions. A well known generalisation of the Lindley distribution is the quasi Lindley (QL) distribution proposed and studied by Shanker and Mishra (2013). Statistically speaking, a random variable $X$ has the QL distribution with parameters $\beta$ and $\theta$ if its probability density function (pdf) and cumulative distribution function (cdf) are respectively, given by

$g(x)=\frac{\theta(\beta+\theta x)}{\beta+1} \mathrm{e}^{-\theta x}, x>0, \beta>-1, \theta>0$

and

$G(x)=1-\frac{\theta(\beta+1+\theta x)}{\beta+1} \mathrm{e}^{-\theta x}, x>0, \beta>-1, \theta>0$.

If $\mathrm{X}$ has the QL distribution with parameters $\beta$ and $\theta$, we write $\mathrm{X} \sim \operatorname{QLD}(\beta, \theta)$.

In recent studies, authors have extended the QL distribution to obtain several distributions. Gui (2014) introduced a generalised QL distribution. Roozegar and Esfandiyari (2015) proposed and studied the statistical properties of McDonald QL distribution. Elgharhy et al. (2017) proposed the transmuted generalised quasi Lindley distribution. Furthermore, transmuted Kumaraswamy QL distribution has been studied by Elgarhy et al. (2018). The exponentiated quasi Lindley (EQL) distribution was proposed by Elbatal et al. (2016). After determining some properties of the distribution, the authors proceeded to showcase its applications. The properties and applications of Weibull quasi Lindley (WQL) distribution were presented by Hasssan et al. (2016). Ghica et al. (2017) introduced the exponentiated power quasi Lindley (EPQL) distribution, highlighting its properties.

An important and widely used method of generalising distributions was introduced by Marshal and Olkin (1997). With this technique, it is possible to add one parameter to a baseline distribution. Let $X$ be a continuous random variable having arbitrary baseline survival function 
$\bar{G}(x)=1-G(x)$, where $G(x)$ is the cdf of $X$. Then the corresponding Marshal-Olkin extended distribution has the survival function

$\bar{F}(x)=\frac{\alpha \bar{G}(x)}{1-\bar{\alpha} \bar{G}(x)},-\infty<x<\infty, \alpha>0, \bar{\alpha}=1-\alpha$.

In (3), $\bar{\alpha}$ is called a tilt parameter. Associated with the survival function (3) are the pdf $(f(x))$ and cdf $(F(x))$ defined by

$f(x)=\frac{\alpha g(x)}{(1-\bar{\alpha} \bar{G}(x))^{2}},-\infty<x<\infty, \alpha>0, \bar{\alpha}=1-\alpha$

and

$F(x)=\frac{\alpha G(x)}{1-\bar{\alpha} \bar{G}(x)},-\infty<x<\infty, \alpha>0, \bar{\alpha}=1-\alpha$.

Sequel to the introduction of the Marshal-Olkin (MO) method, several Marshal-Olkin extended distributions have been introduced. Ghitany et al. (2013), Lepetu et al. (2017) and Hibatullah et al. (2018) introduced the Marshal-Olkin extended Lindley distribution, Marshal-Olkin log-logistic extended Weibull distribution and Marshal-Olkin extended power Lindley distribution respectively. Again, Mansoor et al. (2018) proposed the Marshal-Olkin logistic-exponential distribution.

In this paper, we introduce and study properties of the Marshal-Olkin extended quasi Lindley distribution (MOEQLD). We are motivated to introduce this distribution for two obvious reasons. First, the distribution is indeed one of the useful generalisations of the Lindley distribution and quasi Lindley distribution, which is capable of providing better fits to some data than existing distributions, especially its submodels. Second, autoregressive (AR) minification processes have been defined based on some Marshal-Olkin extended distributions (Jose et al. ,2010; Jose, 2011; Gui, 2013; Krishan et al., 2013; Jayakumar and Babu, 2015). To the best of knowledge of the authors, none of such processes has been proposed in terms of Marshal-Olkin extended Lindley distribution or any of its generalisations. With proven usefulness of Marshal-Olkin extended quasi Lindley distribution (MOEQLD) in data analysis, it will be expedient to discuss its time series applications, especially in the area of AR minification processes.

\section{The proposed distribution}

In this section, we first obtain the survival function (reliability function) of the MOEQLD by using (2) and (3). Consequently, we have

$\bar{F}(x)=R(x)=\frac{\alpha\left(\frac{(\beta+1+\theta x)}{\beta+1} \mathrm{e}^{-\theta x}\right)}{1-(1-\alpha)\left(\frac{\theta(\beta+1+\theta x)}{\beta+1} \mathrm{e}^{-\theta x}\right)}, x>0, \alpha>0, \beta>-1, \theta>0$.

Figure 1 shows the plot of the reliability function of the MOEQLD for several values $\alpha, \beta$ and $\theta$.
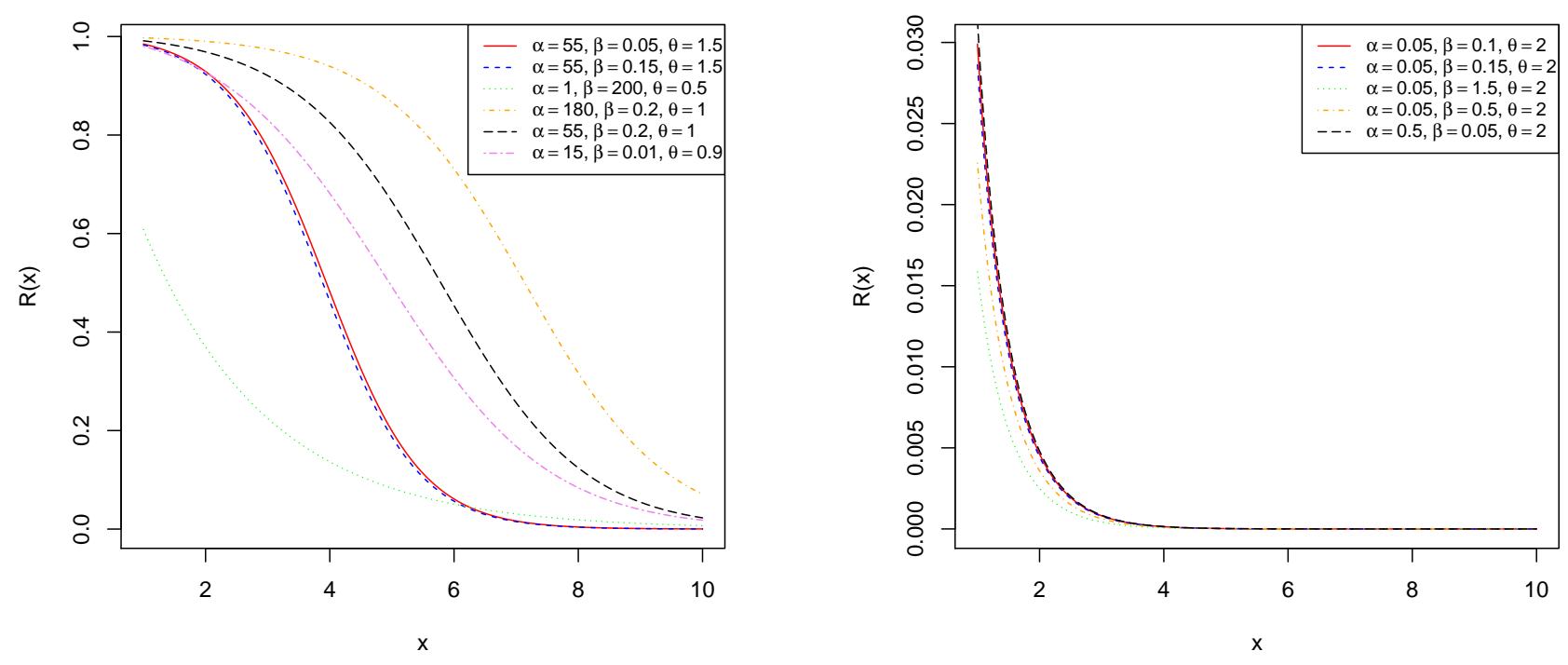

Figure 1: Reliability function of MOEQLD for selected values of $\alpha, \beta$ and $\theta$.

Using (2) and (5), the cdf of the MOEQLD is found to be

$F(x)=\frac{1-\left(\frac{(\beta+1+\theta x)}{\beta+1} \mathrm{e}^{-\theta x}\right)}{1-(1-\alpha)\left(\frac{\theta(\beta+1+\theta x)}{\beta+1} \mathrm{e}^{-\theta x}\right)}, x>0, \alpha>0, \beta>-1, \theta>0$. 

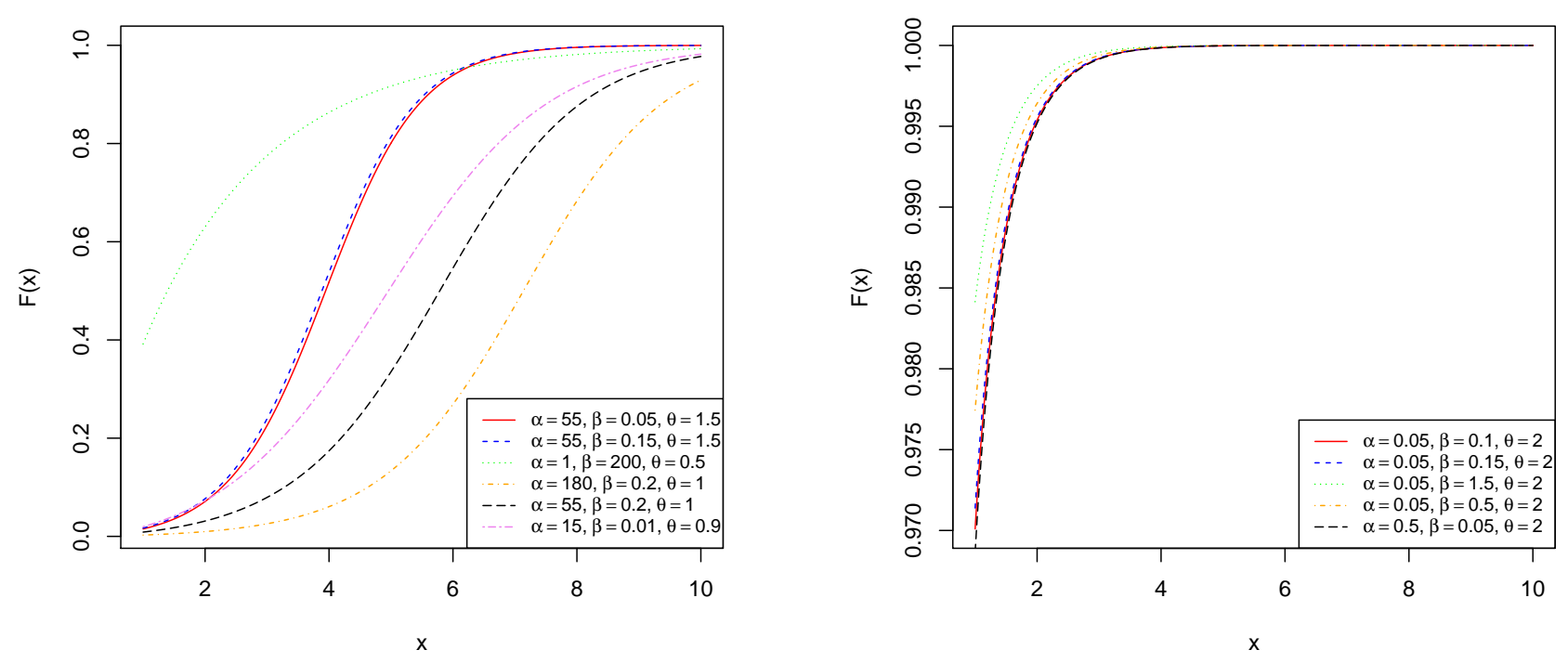

Figure 2: cdf of MOEQLD for selected values of $\alpha, \beta$ and $\theta$.

Furthermore, the pdf of the MOEQLD is of the form

$f(x)=\frac{\left(\frac{\alpha \theta(\beta+\theta x)}{\beta+1} \mathrm{e}^{-\theta x}\right)}{\left(1-(1-\alpha)\left(\frac{(\beta+1+\theta x)}{\beta+1} \mathrm{e}^{-\theta x}\right)\right)^{2}}, x>0, \alpha>0, \beta>-1, \theta>0$. 

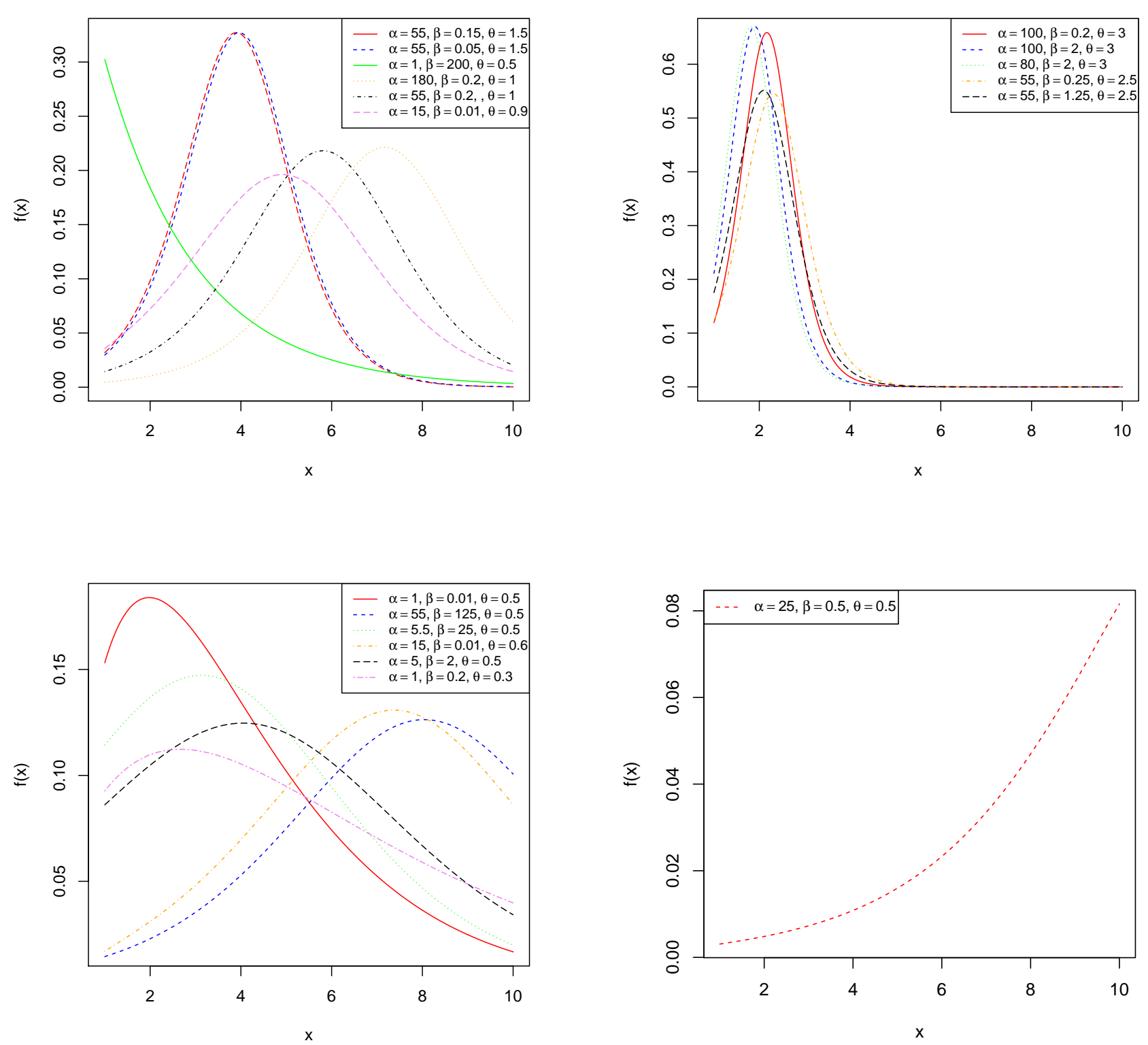

Figure 3: pdf of MOEQLD for selected values of $\alpha, \beta$ and $\theta$.

Dividing (8) by (6) leads to the hazard rate function (hrf)

$h(x)=\frac{\theta(\beta+\theta x)}{(\beta+1+\theta x)\left(1-(1-\alpha)\left(\frac{\theta(\beta+1+\theta x)}{\beta+1} \mathrm{e}^{-\theta x}\right)\right)}, x>0, \alpha>0, \beta>-1, \theta>0$. 


\section{Quantile and random number generation}

Let $X$ be an MOEQL variable with cdf given in (7). The qth quantile of $X$, denoted by $x_{q}$ is the root of the equation

$$
F\left(x_{q}\right)=q \text {, for } q \in(0,1) .
$$

Thus,

$$
\frac{1-\left(\frac{\left(\beta+1+\theta x_{q}\right)}{\beta+1} \mathrm{e}^{-\theta x_{q}}\right)}{1-(1-\alpha)\left(\frac{\theta\left(\beta+1+\theta x_{q}\right)}{\beta+1} \mathrm{e}^{-\theta x_{q}}\right)}=q .
$$

Solving (10) for $x_{q}$ leads to

$x_{q}=-\frac{\beta}{\theta}-\frac{1}{\theta}-\frac{1}{\theta} W_{-1}\left(\frac{(q-1)(\beta+1)}{1-q(1-\alpha)} e^{-(\beta+1)}\right)$,

where $W_{-1}$ denotes the negative branch of the Lambert $\mathrm{W}$ function.

A formula that can be used to generate random numbers from the MOEQLD when the values of its parameters are given, is obtained by substituting $x$ and $u$ into (11) for $x_{q}$ and $q$ respectively. Thus, we have the formula

$x=-\frac{\beta}{\theta}-\frac{1}{\theta}-\frac{1}{\theta} W_{-1}\left(\frac{(u-1)(\beta+1)}{1-u(1-\alpha)} e^{-(\beta+1)}\right)$

where $u \sim U(0,1)$.

\section{Moments and related concepts}

To derive moments of the MOEQLD, we consider expansions of the concerned pdf using (8) and (9) in Cordeiro et al. (2014). Thus, for $\alpha \in(0,1)$ and $\alpha>1$., we have

$f(x)=\sum_{k=0}^{\infty} \rho_{k} h_{k+1}(x)$

and

$f(x)=\sum_{k=0}^{\infty} \omega_{k} h_{k+1}(x)$,

respectively, where $\rho_{k}=\frac{\alpha(-1)^{k}}{k+1} \sum_{j=k}^{\infty}\left(\begin{array}{l}j \\ k\end{array}\right)(j+1) \bar{\alpha}^{j}, k=0,1, \cdots$,

$\omega_{k}=\bar{\alpha}^{-1}\left(1-\bar{\alpha}^{-1}\right), k=0,1, \cdots$ and $h_{k+1}(x)$ is the exponentiated quasi Lindley density with power parameter $k+1$.

Furthermore, if $\alpha \in(0,1)$, the rth non-central moment of $X$ is

$E\left(X^{r}\right)=\sum_{k=0}^{\infty} \rho_{k} E\left(X^{r} h_{k+1}(x)\right)$

In (12), $E\left(X^{r} h_{k+1}(x)\right)$ is the $r t h$ raw moment of exponentiated quasi Lindley distribution with power parameter $k+1$. The expression for $E\left(X^{r} h_{k+1}(x)\right)$ can be easily deduced from (12) in Elbatal et al. (2016). Using the expression and the equation above, we obtain

$E\left(X^{r}\right)=\sum_{k=0}^{\infty} \rho_{k} \psi_{l, m}\left[\frac{\beta \Gamma(r+m+1)}{(\theta(l+1))^{r+m+1}}+\frac{\theta \Gamma(r+m+2)}{(\theta(l+1))^{r+m+2}}\right]$

where

$$
\psi_{l, m}=\sum_{l=0}^{\infty} \sum_{m=0}^{l}(-1)^{l}(k+1)\left(\begin{array}{l}
k \\
l
\end{array}\right)\left(\begin{array}{c}
l \\
m
\end{array}\right)\left(\frac{\theta}{\beta+1}\right)^{m+1}
$$

Similarly, if $\alpha>0$, then

$E\left(X^{r}\right)=\sum_{k=0}^{\infty} \omega_{k} \psi_{l, m}\left[\frac{\beta \Gamma(r+m+1)}{(\theta(l+1))^{r+m+1}}+\frac{\theta \Gamma(r+m+2)}{(\theta(l+1))^{r+m+2}}\right]$

Given that $\alpha \in(0,1)$, the moment generating function of an MOEQL variable $X$ is defined as 


$$
\begin{aligned}
M_{X}(t) & =E\left(e^{t X}\right)=\sum_{r=0}^{\infty} \frac{t^{r}}{r !} E\left(X^{r}\right) \\
& =\sum_{r=0}^{\infty} \frac{t^{r}}{r !} \sum_{k=0}^{\infty} \rho_{k} \psi_{l, m}\left[\frac{\beta \Gamma(r+m+1)}{(\theta(l+1))^{r+m+1}}+\frac{\theta \Gamma(r+m+2)}{(\theta(l+1))^{r+m+2}}\right] .
\end{aligned}
$$

On the other hand, if $\alpha>0$ then

$M_{X}(t)=$

$$
=\sum_{r=0}^{\infty} \frac{t^{r}}{r !} \sum_{k=0}^{\infty} \omega_{k} \psi_{l, m}\left[\frac{\beta \Gamma(r+m+1)}{(\theta(l+1))^{r+m+1}}+\frac{\theta \Gamma(r+m+2)}{(\theta(l+1))^{r+m+2}}\right]
$$

Next we study the effect of values of the parameters of the MOEQLD on the coefficients of skewness and kurtosis of the distribution. The coefficients of skewness and kurtosis are computed using the Bowley skewness (S) and Moors kurtosis (K) formulae defined by

$S=\frac{Q\left(\frac{3}{4}\right)-2 Q\left(\frac{2}{4}\right)+Q\left(\frac{1}{4}\right)}{Q\left(\frac{3}{4}\right)-Q\left(\frac{1}{4}\right)}$

and

$K=\frac{Q\left(\frac{7}{8}\right)-Q\left(\frac{5}{8}\right)+Q\left(\frac{3}{8}\right)-Q\left(\frac{1}{8}\right)}{Q\left(\frac{6}{8}\right)-Q\left(\frac{2}{8}\right)}$,

where $Q($.$) is the quantile function.$

Table 1 comprises the values of $\mathrm{S}$ and $\mathrm{K}$ that corresponds to several sets of values of $\alpha, \beta$ and $\theta$. It can be observed that if $\alpha$ is held constant and $\beta$ and $\theta$ increase, then $\mathrm{S}$ and $\mathrm{K}$ increase. Holding $\beta$ constant and increasing both $\alpha$ and $\theta$ will lead to decrease in $\mathrm{S}$. In this instance, $\mathrm{K}$ is neither an increasing nor a decreasing function. When $\alpha$ and $\beta$ increase and $\theta$ is constant, $\mathrm{S}$ and $\mathrm{K}$ decrease. A Similar result is obtained if $\alpha$ and $\beta$ and $\theta$ decrease. Again, increasing the values of $\alpha, \beta$ and $\theta$ results in decrease in $\mathrm{S}$ and $\mathrm{K}$. Table 1 also indicates that $\mathrm{S}$ can be negative or approximately zero for some values of $\alpha, \beta$ and $\theta$. To this effect, the new distribution can be an alternative distribution to some positively-skewed, negatively-skewed and symmetric distribution.

Table 1: Bowley Skewness (S) and Moors Coefficient of Kurtosis (K) of the MOEQLD for Various Parameter Values

\begin{tabular}{ccccc}
\hline$\alpha$ & $\beta$ & $\theta$ & $\mathrm{S}$ & $\mathrm{K}$ \\
\hline 0.5 & 0.5 & 0.5 & 0.2547 & 1.3271 \\
0.5 & 1 & 1 & 0.2870 & 1.3466 \\
0.5 & 1.5 & 1.5 & 0.3059 & 1.3672 \\
0.5 & 2 & 2 & 0.3168 & 1.3827 \\
0.5 & 2.5 & 2.5 & 0.3234 & 1.3938 \\
0.5 & 3 & 3 & 0.3276 & 1.4018 \\
\hline 1 & 0.5 & 1 & 0.1870 & 1.2556 \\
1.5 & 0.5 & .5 & 0.1476 & 1.2327 \\
2 & 0.5 & 2 & 0.1213 & 1.2247 \\
2.5 & 0.5 & 2.5 & 0.1024 & 1.2227 \\
3 & 0.5 & 3 & 0.0881 & 1.2233 \\
\hline 1 & 1 & 0.5 & 0.2086 & 1.2573 \\
1.5 & 1.5 & 0.5 & 0.1765 & 1.2316 \\
2 & 2 & 0.5 & 0.1532 & 1.2209 \\
2.5 & 2.5 & 0.5 & 0.1354 & 1.2168 \\
3 & 3 & 0.5 & 0.1214 & 1.2159 \\
\hline 1 & 0.1 & 3 & 0.1726 & 1.2613 \\
1.5 & 0.5 & 2.5 & 0.1476 & 1.2327 \\
2 & 0.9 & 2 & 0.1317 & 1.2204 \\
2.5 & 1.3 & 1.5 & 0.1192 & 1.2158 \\
3 & 1.7 & 1 & 0.1087 & 1.2149 \\
\hline 0.1 & 0.5 & 1 & 0.3825 & 1.5966 \\
0.5 & 1 & 1.5 & 0.2870 & 1.3466 \\
0.9 & 1.5 & 2 & 0.2367 & 1.2763 \\
1.3 & 2 & 2.5 & 0.2025 & 1.2458 \\
1.7 & 2.5 & 3 & 0.1773 & 1.2307 \\
\hline 25 & 0.5 & 0.5 & -0.0061 & 1.3025 \\
100 & 0.15 & 0.9 & 0.0020 & 1.2844 \\
\hline & & & &
\end{tabular}

Figure 5 contains the plots of Bowley skewness and Moors kurtosis when $\alpha=0.15$. In Figure 6, we illustrate graphically the relationship between each of $\mathrm{S}$ and $\mathrm{K}$ and $\alpha$. It is certain that none of $\mathrm{S}$ and $\mathrm{K}$ is a linear function of $\alpha$. 


\section{Order statistics}

Order statistics are of utmost importance in reliability and other statistical problems. In this section, the pdf of the kth order statistic for the MOEQLD is determined. Suppose $X_{1}, X_{2}, \cdots, X_{n}$ constitute a random sample from the MOEQLD with cdf $(F(x))$ and pdf $(f(x))$ defined in () and () respectively. Let $X_{1: n}, X_{2: n}, \cdots, X_{n: n}$ denote the corresponding order statistics. The pdf of the kth order statistic $\left(X_{k: n}\right)$

$f_{k: n}(x)=\frac{n !}{(n-k) !(k-1) !} f(x)(F(x))^{k-1}(1-F(x))^{n-k}$

Therefore

$f_{k: n}(x)=\frac{n !}{(n-k) !(k-1) !} \sum_{l=0}^{n-k}\left(\begin{array}{c}n-k \\ l\end{array}\right)(-1)^{l} f(x)(F(x))^{k+l-1}$.

On the basis of the cdf $(G(x))$ and pdf $(g(x))$ of the baseline distribution, we have

$f_{k: n}(x)=\frac{n ! g(x)}{(n-k) !(k-1) !} \sum_{l=0}^{n-k}\left(\begin{array}{c}n-k \\ l\end{array}\right)(-1)^{l} \frac{\alpha^{k+l}(G(x))^{k+l-1}}{(1-(1-\alpha) \bar{G}(x))^{k+l+1}}$.

If $\alpha \in(0,1)$,

$(1-(1-\alpha) \bar{G}(x))^{-(k+l+1)}=\sum_{m=0}^{\infty}\left(\begin{array}{c}k+l+m \\ m\end{array}\right)(1-\alpha)^{m}(\bar{G}(x))^{m}$

and

$(\bar{G}(x))^{m}=(1-G(x))^{m}=\sum_{p=0}^{m}\left(\begin{array}{c}m \\ p\end{array}\right)(-1)^{p}(G(x))^{p}$.

Using (20), (21) and (22), the following result is obtained:

$f_{k: n}(x)=\sum_{m=0}^{\infty} \sum_{p=0}^{m} \sum_{l=0}^{n-k} c_{m p l} f_{E Q L}(x, k+l+p, \theta)$

where

$$
c_{m p l}=\frac{n !\left(\begin{array}{c}
k+l+m \\
m
\end{array}\right)\left(\begin{array}{c}
m \\
p
\end{array}\right)\left(\begin{array}{c}
n-k \\
l
\end{array}\right)(-1)^{l+p} \alpha^{k+l}(1-\alpha)^{m}}{(n-k) !(k-1) !}
$$

and $f_{E Q L}(x, k+l+p, \theta)$ is the exponentiated quasi Lindley (EQL) density with power parameter $k+l+p$.

Additionally, if $\alpha>1$, then

$(1-(1-\alpha) \bar{G}(x))^{-(k+l+1)}=\alpha^{-(k+l+1)}\left(1-\left(1-\alpha^{-1}\right) G(x)\right)^{-(k+l+1)}$

Now,

$\left(1-\left(1-\alpha^{-1}\right) G(x)\right)^{-(k+l+1)}=\sum_{s=0}^{\infty}\left(\begin{array}{c}k+l+s \\ s\end{array}\right)\left(1-\alpha^{-1}\right)^{s}(\bar{G}(x))^{s}$.

Applying (24) and (25) in (20) leads to

$f_{k: n}(x)=\sum_{s=0}^{\infty} \sum_{p=0}^{m} \sum_{l=0}^{n-k} d_{s l} f_{E Q L}(x, k+l+s, \theta)$

where

$$
d_{s l}=\frac{n !\left(\begin{array}{c}
k+l+s \\
s
\end{array}\right)\left(\begin{array}{c}
n-k \\
l
\end{array}\right)(-1)^{l} \alpha^{-1}\left(1-\alpha^{-1}\right)^{s}}{(n-k) !(k-1) !}
$$

and $f_{E Q L}(x, k+l+s, \theta)$ is the EQL density with power parameter $k+l+s$. We can deduce from (23) and (26) that pdf of the kth order statistics of the MOEQLD can be expressed as an infinite linear combination of EQL densities. Thus, it is possible to derive some mathematical properties of the MOEQLD using those of the EQL distribution. 


\section{Estimation}

Let $X_{1}, X_{2}, \cdots, X_{n}$ be a random sample of size $\mathrm{n}$ from MOEQLD with pdf (8). Suppose $\varepsilon=(\alpha, \beta, \theta)$ is the unknown parameter vector. Then the likelihood function is

$$
\begin{aligned}
l(\varepsilon) & =\prod_{i=1}^{n} f\left(x_{i}\right) \\
& =\left(\frac{\alpha \theta}{\beta+1}\right)^{n} \prod_{i=1}^{n}\left(\frac{\beta+\theta x_{i} \mathrm{e}^{-\theta x_{i}}}{\left(1-(1-\alpha)\left(\frac{\left(\beta+1+\theta x_{i}\right)}{\beta+1} \mathrm{e}^{-\theta x_{i}}\right)\right)^{2}}\right) .
\end{aligned}
$$

Therefore, the log-likelihood function is

$$
\begin{aligned}
\ln (\varepsilon) & =n \ln (\alpha)+n \ln (\theta)-n \ln (\beta+1)+\sum_{i=1}^{n} \ln \left(\beta+\theta x_{i}\right)-\theta \sum_{i=1}^{n} x_{i} \\
& -2 \sum_{i=1} \ln \left(1-(1-\alpha)\left(\frac{\left(\beta+1+\theta x_{i}\right)}{\beta+1} \mathrm{e}^{-\theta x_{i}}\right)\right) .
\end{aligned}
$$

Finding and equating the partial derivative of $\ln (\varepsilon)$ with respect to each of $\alpha, \beta$ and $\theta$ to zero, we have

$$
\begin{aligned}
\frac{\partial \ln (\varepsilon)}{\partial \alpha} & =0 \\
\Rightarrow \frac{n}{\alpha}-2 \sum_{i=1}^{n} \frac{\left(\frac{\beta+1+\theta x_{i}}{\beta+1}\right) \mathrm{e}^{-\theta x_{i}}}{\left(1-(1-\alpha)\left(\frac{\beta+1+\theta x_{i}}{\beta+1}\right) \mathrm{e}^{-\theta x_{i}}\right)} & =0, \\
\frac{\partial \ln (\varepsilon)}{\partial \beta} & =0 \\
\Rightarrow-\frac{n}{\beta+1}+\sum_{i=1}^{n} \frac{1}{\beta+\theta x_{i}}-2 \sum_{i=1}^{n} \frac{(1-\alpha) \theta x_{i} \mathrm{e}^{-\theta x_{i}}}{\left(1-(1-\alpha)\left(\frac{\beta+1+\theta x_{i}}{\beta+1}\right) \mathrm{e}^{-\theta x_{i}}\right)(\beta+1)^{2}} & =0 \\
\Rightarrow \frac{n}{\theta}+\sum_{i=1}^{n} \frac{x_{i}}{\beta+\theta x_{i}}-\sum_{i=1}^{n} x_{i}-2 \sum_{i=1}^{n} \frac{(1-\alpha)\left(\beta+\theta x_{i}\right) x_{i} \mathrm{e}^{-\theta x_{i}}}{\partial \theta} & =0 \\
\left(1-(1-\alpha)\left(\frac{\beta+1+\theta x_{i}}{\beta+1}\right) \mathrm{e}^{-\theta x_{i}}\right)(\beta+1) & =0
\end{aligned}
$$

To find the maximum likelihood estimates of the unknown parameters, we solve (28), (29) and (30) simultaneously. Since these equations cannot be solved analytically, a numerical approach with iterative procedures such as the Newton Ralphson algorithm is required. Interestingly, some packages in $\mathrm{R}$ can be used to obtain the estimates.

Under certain regularity conditions, when $n \rightarrow \infty$, the distribution of $\hat{\varepsilon}$ can be approximated by a multivariate normal $N_{3}\left(0, J(\hat{\varepsilon})^{-1}\right)$ distribution to construct confidence intervals for the parameters. Here, $J(\hat{\varepsilon})$ is the total observed information matrix evaluated at $\hat{\varepsilon}$. For simplicity, the matrix $J(\hat{\varepsilon})$ is given by

$J(\hat{\varepsilon})=\left(\begin{array}{lll}a_{11} & a_{12} & a_{13} \\ a_{21} & a_{22} & a_{23} \\ a_{31} & a_{32} & a_{33}\end{array}\right)$. 
The elements of the matrix are defined as follows

$$
\begin{aligned}
& a_{11}=-\left.\frac{\partial^{2} \ln (\varepsilon)}{\partial \alpha^{2}}\right|_{\varepsilon=\hat{\varepsilon}} \\
& =\frac{n}{\hat{\alpha}^{2}}-2 \sum_{i=1}^{n}\left(\frac{\left(\frac{\hat{\beta}+1+\hat{\theta} x_{i}}{\hat{\beta}+1}\right) \mathrm{e}^{-\hat{\theta} x_{i}}}{1-(1-\hat{\alpha})\left(\frac{\hat{\beta}+1+\hat{\theta} x_{i}}{\hat{\beta}+1}\right) \mathrm{e}^{-\hat{\theta} x_{i}}}\right)^{2}, \\
& a_{12}=a_{21}=-\left.\frac{\partial^{2} \ln (\varepsilon)}{\partial \alpha \partial \beta}\right|_{\varepsilon=\hat{\varepsilon}} \\
& =\frac{2}{(\hat{\beta}+1)^{2}} \sum_{i=1}^{n} \frac{\hat{\theta} x_{i} \mathrm{e}^{-\hat{\theta} x_{i}}}{\left(1-(1-\hat{\alpha})\left(\frac{\hat{\beta}+1+\hat{\theta} x_{i}}{\hat{\beta}+1}\right) \mathrm{e}^{-\hat{\theta} x_{i}}\right)^{2}}, \\
& a_{13}=a_{31}=-\left.\frac{\partial^{2} \ln (\varepsilon)}{\partial \alpha \partial \theta}\right|_{\varepsilon=\hat{\varepsilon}} \\
& =\frac{2}{\hat{\beta}+1} \sum_{i=1}^{n} \frac{\left(\hat{\beta}+\hat{\theta} x_{i}\right) x_{i} \mathrm{e}^{-\hat{\theta} x_{i}}}{\left(1-(1-\hat{\alpha})\left(\frac{\hat{\beta}+1+\hat{\theta} x_{i}}{\hat{\beta}+1}\right) \mathrm{e}^{-\hat{\theta} x_{i}}\right)^{2}}, \\
& a_{22}=-\left.\frac{\partial^{2} \ln (\varepsilon)}{\partial \beta^{2}}\right|_{\varepsilon=\hat{\varepsilon}} \\
& =-\frac{2}{(\hat{\beta}+1)^{2}}+\sum_{i=1}^{n} \frac{1}{\left(\hat{\beta}+\hat{\theta} x_{i}\right)^{2}} \\
& -\frac{2}{(\hat{\beta}+1)^{4}} \sum_{i=1}^{n} \frac{(1-\hat{\alpha}) \hat{\theta} x_{i}\left(1+2(\hat{\beta}+1)\left(1-(1-\hat{\alpha})\left(\frac{\hat{\beta}+1+\hat{\theta} x_{i}}{\hat{\beta}+1}\right) \mathrm{e}^{-\hat{\theta} x_{i}}\right)\right) \mathrm{e}^{-\hat{\theta} x_{i}}}{\left(1-(1-\hat{\alpha})\left(\frac{\hat{\beta}+1+\hat{\theta} x_{i}}{\hat{\beta}+1}\right) \mathrm{e}^{-\hat{\theta} x_{i}}\right)^{2}}, \\
& a_{23}=a_{32}=-\left.\frac{\partial^{2} \ln (\varepsilon)}{\partial \beta \partial \theta}\right|_{\varepsilon=\hat{\varepsilon}} \\
& =\sum_{i=1}^{n} \frac{x_{i}}{\left(\hat{\beta}+\hat{\theta} x_{i}\right)^{2}}+\frac{2}{(\hat{\beta}+1)^{2}} \sum_{i=1}^{n} \frac{(1-\hat{\alpha}) x_{i}\left(1-\hat{\theta}-\hat{\theta} x_{i}-(1-\hat{\alpha}) \mathrm{e}^{-\hat{\theta} x_{i}}\right) \mathrm{e}^{-\hat{\theta} x_{i}}}{\left(1-(1-\hat{\alpha})\left(\frac{\hat{\beta}+1+\hat{\theta} x_{i}}{\hat{\beta}+1}\right) \mathrm{e}^{-\hat{\theta} x_{i}}\right)^{2}}
\end{aligned}
$$

and

$$
\begin{aligned}
a_{33} & =-\left.\frac{\partial^{2} \ln (\varepsilon)}{\partial \theta^{2}}\right|_{\varepsilon=\hat{\varepsilon}} \\
& =\frac{n}{\hat{\theta}^{2}}+\sum_{i=1}^{n} \frac{x_{i}^{2}}{\left(\hat{\beta}+\hat{\theta} x_{i}\right)^{2}} \\
& +\frac{2}{(\hat{\beta}+1)^{2}} \sum_{i=1}^{n} \frac{(1-\hat{\alpha}) x_{i}^{2}\left((\hat{\beta}+1)\left(1-\hat{\beta}-\hat{\theta} x_{i}\right)-(1-\hat{\alpha}) \mathrm{e}^{-\hat{\theta} x_{i}}\right) \mathrm{e}^{-\hat{\theta} x_{i}}}{\left(1-(1-\hat{\alpha})\left(\frac{\hat{\beta}+1+\hat{\theta} x_{i}}{\hat{\beta}+1}\right) \mathrm{e}^{-\hat{\theta} x_{i}}\right)^{2}} .
\end{aligned}
$$

The inverse matrix $J(\hat{\varepsilon})^{-1}$ is the estimated variance-covariance matrix for the unknown parameter vector $\varepsilon$. Mathematically,

$$
J(\hat{\varepsilon})^{-1}=\left(\begin{array}{ccc}
\widehat{\operatorname{Var}(\hat{\alpha})} & \widehat{\operatorname{Covar}(\hat{\alpha}, \hat{\beta})} & \widehat{\operatorname{Covar}(\hat{\alpha}, \hat{\theta})} \\
\operatorname{Covar}(\hat{\alpha}, \hat{\beta}) & \widehat{\operatorname{Var}(\hat{\beta})} & \operatorname{Covar}(\hat{\beta}, \hat{\theta}) \\
\operatorname{Covar}(\hat{\alpha}, \hat{\theta}) & \operatorname{Covar(\hat {\beta },\hat {\theta })} & \widehat{\operatorname{Var}(\hat{\theta})}
\end{array}\right) .
$$

Thus, the approximate $100(1-v) \%$ two-sided confidence intervals for $\alpha, \beta$ and $\theta$ are, respectively, given by $\hat{\alpha} \pm Z_{\frac{v}{2}} \sqrt{\widehat{\operatorname{Var}(\hat{\alpha})}}, \hat{\beta} \pm$ $Z_{\frac{v}{2}} \sqrt{\widehat{\operatorname{Var}(\hat{\beta})}}$ and $\hat{\theta} \pm Z_{\frac{v}{2}} \sqrt{\widehat{\operatorname{Var}(\hat{\theta})}}$. Here, $Z_{\frac{v}{2}}$ is $\frac{v}{2}$ th upper percentile of the standard normal distribution.

\section{MOEQLD autoregressive minification processes}

A good number of time series are non-Guassian with Markovian structure. The introduction of autoregressive models with minification structure is one of the steps taken to meet the need for time series models with non-Guassian marginals. Recently, several Marshall-Olkin extended distributions and their associated AR minification processes have been introduced. In this section, we introduce the MOEQLD Autoregressive Minification Processes. Consider an AR (1) minification process defined by 
$X_{n}= \begin{cases}\varepsilon_{n} & \text { with probability } \gamma, \\ \min \left(X_{n-1}, \varepsilon_{n}\right) & \text { with probability } 1-\gamma .\end{cases}$

In (31), $\gamma \in(0,1)$ and $\left\{\varepsilon_{n}\right\}$ is a sequence of independent and identically distributed random variables independent of $\left\{X_{n}\right\}$.

Theorem 7.1. For the minification process (31), $\left\{X_{n}\right\}$ is a stationary Markovian process with the marginal as the MOEQLD( $\gamma, \beta$, $\left.\theta\right)$ if and only if $\left\{\varepsilon_{n}\right\}$ has a $Q L D(\beta, \theta)$.

Proof. From (31), we have

$$
\bar{F}_{X_{n}}(x)=\gamma \bar{F}_{\varepsilon_{n}}(x)+(1-\gamma) \bar{F}_{X_{n-1}}(x) \bar{F}_{\varepsilon_{n}}(x)
$$

Under stationarity, we obtain

$\bar{F}_{X}(x)=\frac{\gamma \bar{F}_{\varepsilon_{n}}(x)}{1-(1-\gamma) \bar{F}_{\varepsilon_{n}}(x)}$.

Substituting the survival function $\bar{F}_{\varepsilon_{n}}(x)$ of $\varepsilon$ into (25) leads to

$\bar{F}_{X}(x)=\frac{\gamma\left(\frac{(\beta+1+\theta x)}{\beta+1} \mathrm{e}^{-\theta x}\right)}{1-(1-\gamma)\left(\frac{(\beta+1+\theta x)}{\beta+1} \mathrm{e}^{-\theta x}\right)}$.

It can be easily deduce that $\bar{F}_{X}(x)$ is the survival function of an $\operatorname{MOEQLD}(\gamma, \beta, \theta)$.

Conversely, let us assume that $X_{n}$ follows an $\operatorname{MOEQLD}(\gamma, \beta, \theta)$. Assuming stationarity, the following equation is obtained from (31)

$\bar{F}_{\varepsilon_{n}}(x)=\frac{\gamma \bar{F}_{X}(x)}{\gamma+(1-\gamma) \bar{F}_{X}(x)}$.

After substituting the survival function of $\operatorname{MOEQLD}(\gamma, \beta, \theta)$ for $\bar{F}_{X}(x)$ in (34), we get

$\bar{F}_{\varepsilon_{n}}(x)=\frac{(\beta+1+\theta x)}{\beta+1} \mathrm{e}^{-\theta x}$,

which is the survival function of the $\operatorname{QLD}(\beta, \theta)$.

Next, we discuss a more general AR(1) structure that allows for probabilistic selection of process values, innovations and combinations of both process values and innovations. The AR(1) structure is given in Theorem 7.2.

Theorem 7.2. Consider the AR(1) structure

$X_{n}= \begin{cases}X_{n} & \text { with probability } \gamma_{2} \\ \varepsilon_{n} & \text { with probability } \gamma_{1}\left(1-\gamma_{2}\right), \\ \min \left(X_{n-1}, \varepsilon_{n}\right) & \text { with probability }\left(1-\gamma_{1}\right)\left(1-\gamma_{2}\right),\end{cases}$

where $\left\{\varepsilon_{n}\right\}$ is a sequence of independent and identically distributed random variables independent of $\left\{X_{n}\right\}$. Then $\left\{X_{n}\right\}$ is a stationary Markovian process with MOEQL marginal if and only if $\left\{\varepsilon_{n}\right\}$ has a quasi Lindley distribution.

Proof. From the given AR(1) structure, we obtain

$\bar{F}_{X_{n}}(x)=\gamma_{2} \bar{F}_{X_{n-1}}(x)+\gamma_{1}\left(1-\gamma_{2}\right) \bar{F}_{\varepsilon_{n}}(x)+\left(1-\gamma_{1}\right)\left(1-\gamma_{2}\right) \bar{F}_{X_{n-1}}(x) \bar{F}_{\varepsilon_{n}}(x)$.

To conclude this proof, we proceed as in the proof of Theorem 7.1.

\section{Application}

In this section, we illustrate the applicability the MOEQLD using a real dataset. The data, which contain the salaries (in dollars) of 818 professional baseball players for the year 2018 have been reported and modelled by Oluyede et al. (2016).

In fitting the MOEQLD to the data, we employ the maximum likelihood estimation approach using the fitdist function in R. -2lnL, Akaike Information Criterion $(A I C)$ and Bayesian Information Criterion $(B I C)$ are used to compare fits of the distribution with the fits of the associated submodels, namely, the MOELD, QLD and LD. Here,

$$
\mathrm{AIC}=2 \mathrm{p}-2 \ln \mathrm{L} \text {, and } \mathrm{BIC}=\mathrm{p} \ln (\mathrm{n})-2 \ln \mathrm{L},
$$

where $\mathrm{p}$ is the number of parameters contained in a model, $\mathrm{n}$ is the sample size, $\ln \mathrm{L}$ is the value of the log-likelihood function that corresponds to the maximum likelihood estimate(s) of the parameter(s) of the given model. A distribution with minimum values of -2lnL, AIC and BIC will be taken to be most suitable distribution among the four distributions fitted to the data.

In Table 2, maximum likelihood estimates (MLEs) of the model parameters, the corresponding standard error estimates(SEE)and values of -lnL, AIC and BIC are presented. 
Table 2: Parameter Estimates and Corresponding Values of Model Selection Criteria

\begin{tabular}{cccccc}
\hline Distribution & MLE & SEE & $-\operatorname{lnL}$ & AIC & BIC \\
\hline MOEQLD $(\alpha, \beta, \theta)$ & $\hat{\alpha}=0.1926$ & 0.0444 & 1703.416 & 3412.832 & 3426.952 \\
& $\hat{\lambda}=2.6808$ & 2.2341 & & & \\
& $\hat{\theta}=0.1625$ & 0.0203 & & & \\
\hline $\operatorname{MOELD}(\alpha, \theta)$ & $\hat{\alpha}=0.0599$ & 0.0143 & 1727.038 & 3458.077 & 3467.491 \\
& $\hat{\theta}=0.1752$ & 0.0220 & & & \\
\hline $\operatorname{QLD}(\beta, \theta)$ & $\hat{\beta}=135.2934$ & 433.0882 & 1784.685 & 3573.37 & 3582.784 \\
& $\hat{\theta}=0.3091$ & 0.0129 & & & \\
\hline $\operatorname{L}(\theta)$ & $\hat{\theta}=0.5099$ & 0.0130 & 1919.938 & 3841.876 & 3846.583 \\
\hline
\end{tabular}

It can be easily deduced that the minimum values of the criteria adopted in this section are all associated with the MOEQLD. As a consequence, we conclude that the MOEQLD is the best among the four models fitted to the data. Figure 7 contains the plots of the estimated pdfs and cdfs for the data.

\section{References}

[1] L. Benkhelifa," The Marshall-Olkin extended generalised Lindley distribution: Properties and applications", Communication in Statistics-Simualtion and Computation, 46(10), (2017), 8306-8330.

[2] G. M. Cordeiro, A. J. Lemonte, M. M. Ortega (2014), 'The Marshall-Olkin family of distributions: Mathematical properties and new models", Journal of Statistical Theory and Practice, Doi: 10.1080/15598608.2013.802659.

[3] I. Elbatal, L. S. Diab, M. Elgarhy, "Exponentiated quasi Lindley distribution”, International Journal of Reliability and Applications, 17(1), (2016), 1-19.

[4] M. Elgarhy, I. Elbatal, L. S. Diab, H. K. Hwas, A. W. Shawki, "Transmuted generalised quasi Lindley distribution”, International Journal of Scientific Engineering and Science, 1(7), (2017), 1-8.

[5] M. Elgarhy, I. Elbatal, M. A. U. Haq, A. S. Hassan, ”Transmuted Kumaraswamy quasi Lindley distribution with applications”, Annals of Data Science, 5(4), (2018), 565-581

[6] M. Ghica, N. D. Poesina, I. Prasacu, "Exponentiated power quasi Lindley distribution. submodels and some properties", Review of the Air Force Academy, 2(34), (2017), 75-84.

[7] M. E. Ghitany, M. Al-Buraies, D. K. Al-Mutari, "Marshall-Olkin extended Lindley distribution and its application", International Journal of Applied Mathematics, 25(5), (2012),709-721.

[8] W. Gui, "Marshall-Olkin extended log-logistic distribution and its minification processes", 7(80), (2013), 3947-3961.

[9] A. S. Hassan, I. Elbatal, S. E. Hameda, Weibull quasi Lindley distribution applications to lifetime data, International Journal of Applied Mathematics and Statistics, 55(3),(2016), 63-80.

[10] R. Hibatullah, Y. Widyaningsih, S. Abdullah, "Marshall-Olkin extended power Lindley distribution with application", J. Ris. and Ap. Mat., 2(2), (2018),

[11] K. Jayakumar, and M. G. Babu, "Some generalisations of Weibull distribution and related processes", Journal of Statistical Theory and Applications, 14(4), (2015), 425-434.

[12] K. K. Jose, "Marshall-Olkin family of distributions and their applications in reliability theory, time series modelling and stress-strenght analysis", Int. Statistical Inst: Proc. 58th World Statistical Congress, (2011), 3918-3923.

[13] K. K. Jose, S. R. Naik, M. M. Ristic, "Marshall-Olkin q-Weibull distribution and max-min processes”, Stat papers, 51(2010),837-851.

[14] E. Krishnan, K. K. Jose, and M. M. Ristic, "Applications of Marshall-Olkin Frétchet distribution", Communication in Statistics-Simulation and Computation, 42(2013), 76-89.

[15] L. Lepetu, B. O. Oluyede, B. Makubate, S. Foya,and P. Mdlongwa, "Marshall-Olkin log-logistic extended Weibull distribution: Theory, Properties and applications", Journal of Data Science, 15 (2017), 691-722.

[16] D. V. Lindley, "Fiducial distributions and Bayes' theorem", Journal of the Royal Statistical Society, Series A (20),(1958), 102-107.

[17] M. Mansoor, M. H. Tahir, G. M. Cordeiro, S. B. Provost, and A. Alzaatreh, "The Marshall-Olkin logistic-exponential distribution", Communication in Statistics-Theory and Methods, https://doi.org/10.1080/03610926.2017.1414254.

[18] A. W. Marshall, and I. Olkin, "A new method of adding a parameter to a family of distributions with application to the exponential and Weibull families", Biometrika, 84(3), (1997), 641-652.

[19] B. O. Oluyede, and S. Rajasooriya, "The Mc-Dagum distribution and its statistical properties with applications", Asian Journal of Mathematics and Applications, 2013 (2013), 1-16

[20] R. Roozegar, F. Esfandiyari, "The McDonald quasi Lindley distribution and its statistical properties with applications", J. Stat. Appl. Proc., 4(3), (2015), 375-386.

[21] R. Shanker, A. Mishra, "A quasi Lindley distribution”, African Journal of Mathematics and Computer Science Research, 6(4), (2013), 64-71. 

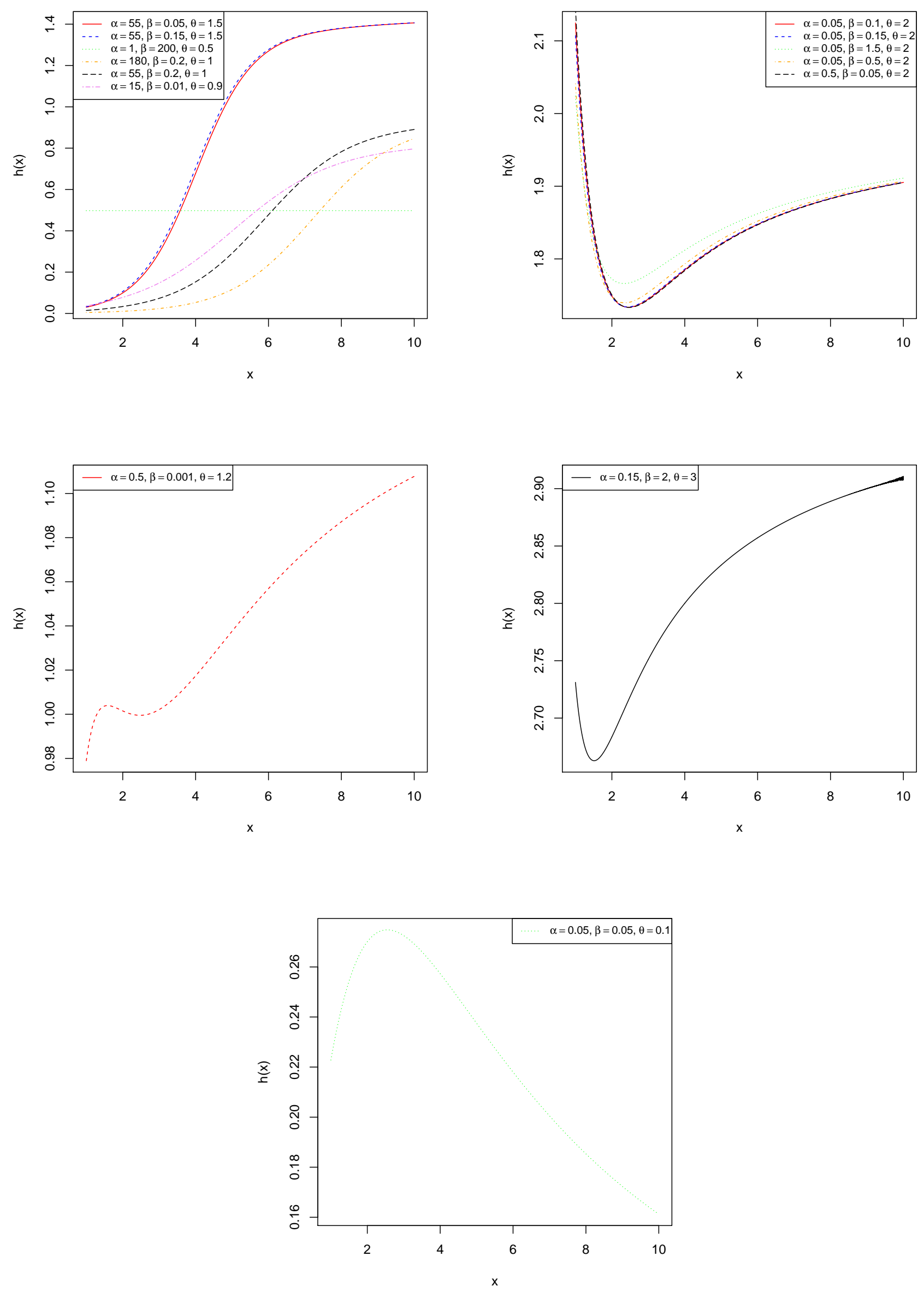

Figure 4: hrf of MOEQLD for selected values of $\alpha, \beta$ and $\theta$. 

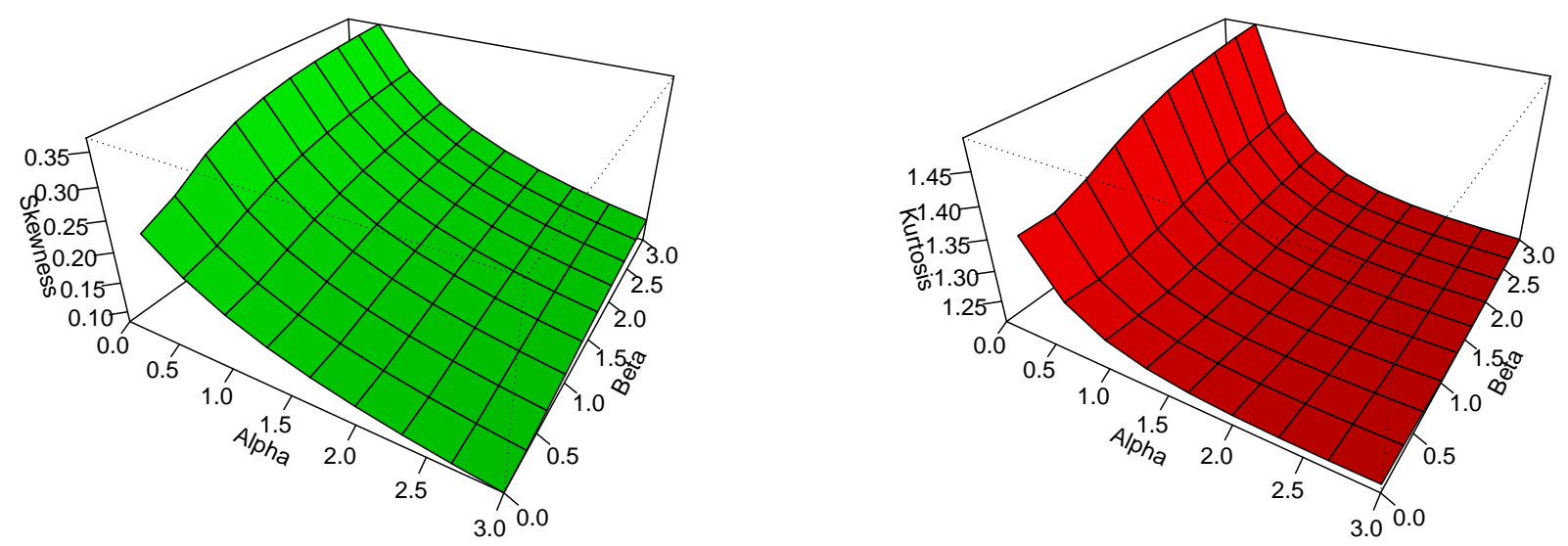

Figure 5: Bowley Skewness and Moors Kurtosis Plots for Some Values of $\alpha$ and $\beta$ and $\theta=0.15$
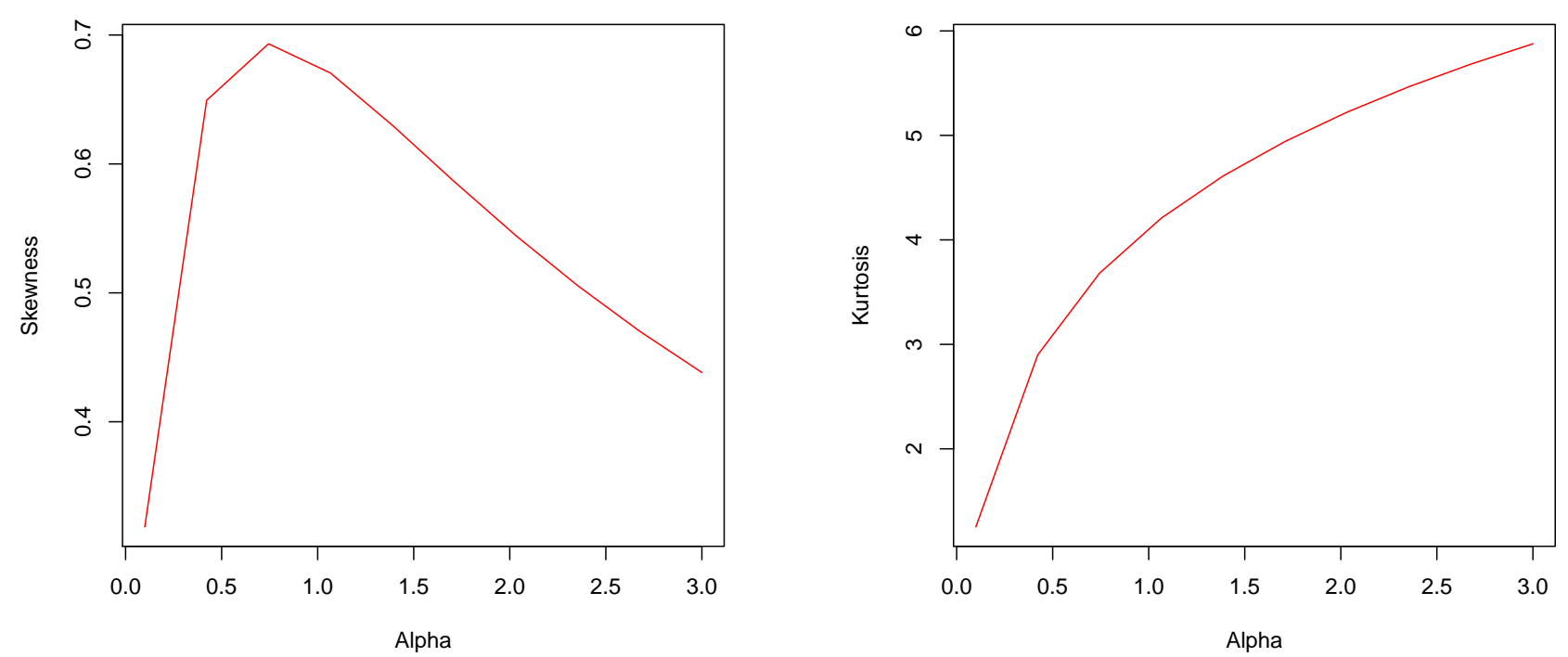

Figure 6: Bowley Skewness and Moors Kurtosis Plots for Some Values of $\alpha$ and $\beta=1.5$ and $\theta=0.5$ 
International Journal of Advanced Statistics and Probability

41
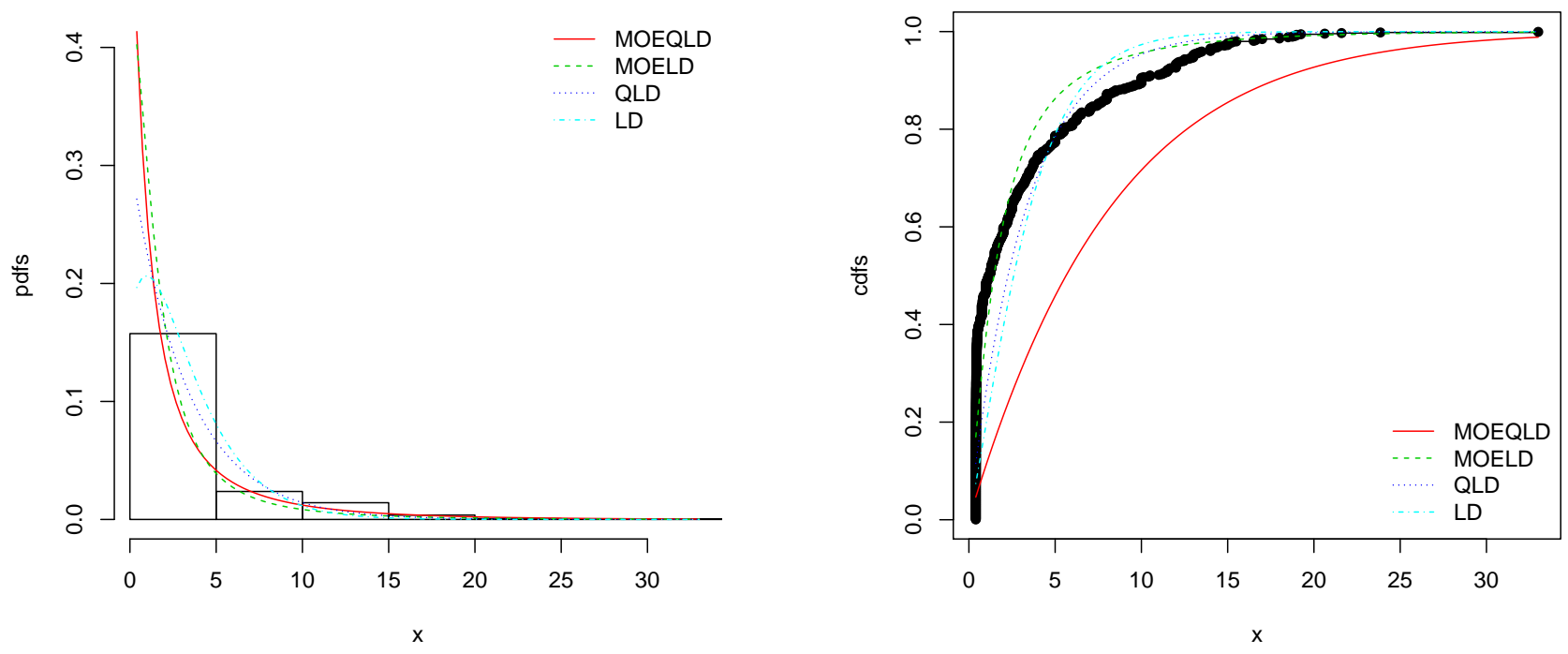

Figure 7: Estimated pdf and cdfs Plots 